\title{
FÍSTULA PANCREATOPLEURAL POR PSEUDOCISTO PANCREÁTICO: RELATO DE UM CASO DE EVOLUÇÃO INCOMUM
}

\section{PANCREATO PLEURAL FISTULA DUE TO PANCREATIC PSEUDOCYST: REPORT OF A CASE OF UNUSUAL EVOLUTION}

Gabriela Camilo Teixeira ${ }^{1}$; Maurício Campanelli Costas ${ }^{1}$; Felipe Emanuel Fuhro ${ }^{1}$; Aline Alves Casteletti ${ }^{1}$; Gabriel Lopes de Lima ${ }^{1}$; Esaú Furini Ferreira Barros ${ }^{1}$; Jaques Waisberg ${ }^{1}$; Edgard Porto de Oliveira Pontes$^{2}$; André Marini Menini1 ${ }^{1,2}$.

\section{RESUMO}

Introdução: $O$ pseudocisto pancreático pode ocorrer, em geral, por complicações da pancreatite aguda. Geralmente se desenvolve entre quatro a seis semanas após o início da pancreatite e ocorre entre 16 a $50 \%$ dos casos de pancreatite aguda e 20-40\% na crônica. Em aproximadamente apenas 0,4\% dos casos de pancreatite pode ocorrer comunicação com a cavidade pleural, formando uma fistula pancreatopleural, complicação rara e pouco descrita na literatura. O diagnóstico muitas vezes é dificil por conta de sua apresentação clínica atípica. Em relação ao tratamento, não existe um consenso definido. Em geral, pode-se realizar tratamento clínico. Aproximadamente $40 \%$ dos casos respondem à terapia clínica. Em casos refratários, ou com rotura de pseudocistos, opta-se pela conduta cirúrgica. Relato do caso: Nesse estudo, será relatado um caso raro de fistula pancreatopleural por um pseudocisto traumático, com tratamento cirúrgico efetivo. Conclusão: O relato em questão ilustra um caso incomum na literatura, com relevância na prática cirúrgica.

Palavras-chave: Fístula. Pâncreas. Pseudocisto Pancreático.

\section{ABSTRACT}

Introduction: Pancreatic pseudocyst can occur, in general, due to complications of acute pancreatitis. It usually develops between four to six weeks after the onset of pancreatitis and occurs in between 16 to $50 \%$ of cases of acute pancreatitis and between 20 and $40 \%$ in chronic pancreatitis. In approximately only $0.4 \%$ of the cases of pancreatitis, communication with the pleural cavity can occur, forming a pancreatico-pleural fistula, a rare complication and little described in the literature. The diagnosis is often difficult due to its atypical presentation. Regarding treatment, there is no definite consensus. In general, clinical treatment can be performed. Approximately $40 \%$ of cases respond to clinical therapy. In refractory cases or with rupture of pseudocysts, surgical management is chosen. Case report: In this study, a rare case of pancreato pleural fistula due to a traumatic pseudocyst will be reported, with effective surgical treatment. This report illustrates an unusual case in the literature, with relevance in surgical practice. Conclusion: This report illustrates an unusual case in the literature, with relevance in surgical practice.

Keywords: Fistula. Pancreas. Pancreatic Cyst.

\section{INTRODUÇÃO}

O pseudocisto pancreático pode ocorrer, em geral, por complicações da pancreatite aguda ${ }^{1}$. Geralmente se desenvolve entre quatro a seis semanas após o início da pancreatite e ocorre entre 16 a $50 \%$ dos casos de pancreatite aguda e 20$40 \%$ na crônica ${ }^{2}$.

Pela classificação de Atlanta, ele é definido como uma coleção encapsulada de fluido com uma parede inflamatória bem definida, geralmente fora do pâncreas, sem necrose.
$\mathrm{Na}$ imagem, apresenta-se com distribuição homogênea com densidade fluida, bem circunscrito, com componente estritamente líquido de paredes bem definidas e encapsulado3.

Em aproximadamente apenas 0,4\% dos casos de pancreatite pode ocorrer comunicação com a cavidade pleural, formando uma fistula pancreatopleural, complicação rara e pouco descrita na literatura ${ }^{4}$.

A formação de tais fistulas em geral está associada a pacientes do sexo

\footnotetext{
${ }^{1}$ Faculdade de Medicina do ABC - Cirurgia Geral e do Aparelho Digestivo - Santo André - SP - Brasil;

${ }^{2}$ Faculdade de Medicina do ABC - Cirurgia Torácica - Santo André - SP - Brasil
} 
masculino (4:1) na faixa dos 45 anos com histórico de pancreatite crônica, em especial de etiologia alcóolica, representando uma mortalidade em torno de $5 \%{ }^{5}$.

Pode-se apresentar como uma comunicação diretamente do ducto pancreático principal até a cavidade pleural ou através de um pseudocisto ${ }^{5}$.

O diagnóstico muitas vezes é dificil por conta de sua apresentação atípica, sendo a manifestação clínica mais comum o desenvolvimento de sintomas respiratórios, em especial evoluindo com derrame pleural. A dor abdominal não é comum e a investigação diagnóstica pode ser feita com uma ressonância de abdome e uma pancreatografia que evidenciam o trajeto fistuloso 5 .

Em relação ao tratamento, não existe um consenso definido. Em geral, pode-se realizar tratamento clínico com somatostatina, jejum via oral e nutrição parenteral total, além da drenagem torácica por cerca de 2 a 3 semanas; ou a colocação de stents na via pancreática pela CPRE. Aproximadamente $\quad 40 \%$ dos casos respondem à terapia clínica. Em casos refratários, ou com rotura de pseudocistos, opta-se pela conduta cirúrgica6,7.

Nesse estudo, será relatado um caso raro de fistula pancreatopleural por um pseudocisto traumático, com tratamento cirúrgico efetivo.

\section{RELATO DO CASO}

M.R.S.F., 47 anos, sem comorbidades, etilismo ou tabagismo, admitida em serviço público, em 10/01/2021, com queixa de dor abdominal em hipocôndrio esquerdo há 3 meses, com piora na última semana. Relata emagrecimento de $10 \mathrm{~kg}$ no período, sem demais queixas. Nega episódios prévios de pancreatite, porém relata agressão física há 8 anos, quando descobriu existência de cisto pancreático, no entanto, nunca realizou seguimento do mesmo.

Em ultrassonografia de abdome, ausência de cálculos em vesícula biliar e ausência de dilatação de vias biliares. Realizados exames para investigação com tomografia computadorizada de abdome, ressonância magnética e colangiorressonância, que evidenciaram um pseudocisto pancreático de dimensões $100 \mathrm{x}$ $52 \times 98 \mathrm{~mm}$ na topografia da cauda pancreática, fazendo corpo com rim esquerdo e baço, sem planos de clivagem com essas estruturas, com cápsula de pseudocisto integra (Figuras 1A e 1B).

Por conta do estado nutricional da paciente, foi optado por dieta imunomoduladora pré-operatória para posterior abordagem cirúrgica. Durante esse período, paciente procurou novamente pronto socorro por dispneia progressiva intensa, com necessidade de suporte ventilatório, em 28/01/2021. Realizou exames complementares, e em radiografia de tórax, visualizado velamento de hemitórax esquerdo e desvio de traqueia e mediastino contralateral (Figura 1C). Optado assim por drenagem pleural à esquerda, com saída de $900 \mathrm{ml}$ xantocrômico e melhora expressiva do quadro clínico da paciente.

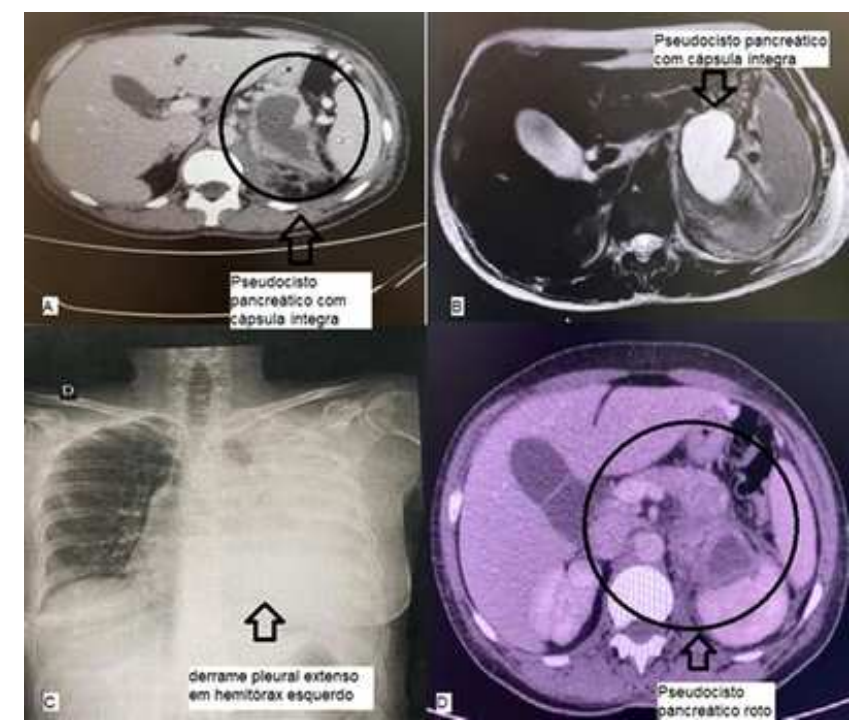

Figura 1. A: Tomografia de abdome inicial com contraste endovenoso na fase portal evidenciando pseudocisto pancreático com cápsula integra. B: Ressonância magnética inicial evidenciando pseudocisto pancreático com cápsula íntegra. C: Radiografia de tórax com derrame pleural extenso, após rotura de pseudocisto fistulizado para hemitórax esquerdo. D: Tomografia de abdome com contraste endovenoso na fase portal evidenciando pseudocisto roto.

Realizada nova tomografia de tórax e abdome, que evidenciou pseudocisto e formação de fistula pancreatodiafragmática, motivo pelo qual ocorreu derrame pleural extenso à esquerda que levou a paciente a procurar atendimento médico novamente antes da data preconizada do procedimento cirúrgico (Figura 1D).

No exame bioquímico coletado do líquido pleural, apresentava amilase 3423, 
citologia oncótica negativa e demais parâmetros bioquímicos sem alterações, reiterando ainda mais o diagnóstico de fistula pancreática para cavidade pleural.

Para melhor investigação, foram solicitadas ecoendoscopia e pancreatografia. A ecoendoscopia constatou parênquima pancreático com ecotextura preservada, presença de extensa imagem ovalada em topografia de corpo distal e cauda de pâncreas, homogênea, com contornos bem delimitados, medindo 96 x $52 \mathrm{~mm}$ nos maiores eixos e paredes espessadas; ducto pancreático principal com calibre de $2 \mathrm{~mm}$ na porção cefálica. Vesícula biliar com parede regular e múltiplas imagens hiperecoicas, medindo menos de $2 \mathrm{~mm}$ cada, compativeis com microlitiase.

Em pancreatografia, nota-se ducto pancreático sem dilatações, porém com extravasamento de contraste em projeção de corpo para cauda do pâncreas. Realizada esfincterocotomia com ENDOCUT I e drenagem do contraste, com posterior passagem de prótese pancreática.

Dessa forma, com os exames complementares, foi confirmado o diagnóstico de fistula pancreaticopleural. Assim, após estabilização do quadro pulmonar, em 04/02/2021, foi submetida a pancreatectomia corpo caudal com esplenectomia e frenorrafia posteromedial a esquerda + colecistectomia por pseudocisto pancreático fistulizado para hemitórax esquerdo (Figura 2).

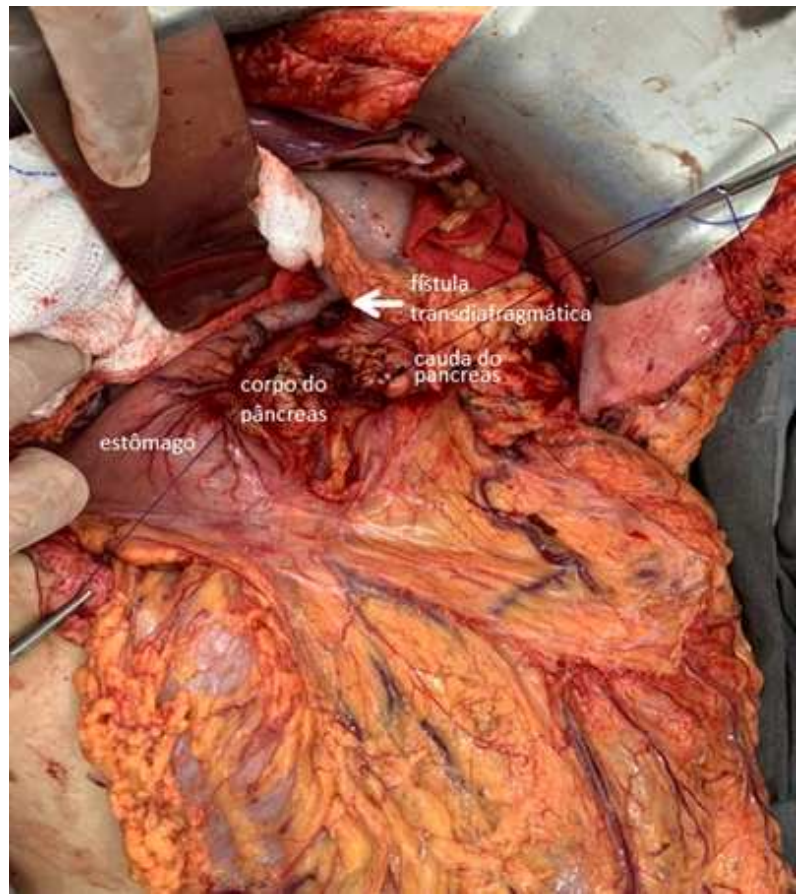

Figura 2: Intraoperatório.
Em intraoperatório, visualizada fistula diafragmática de aproximadamente $2 \mathrm{~cm}$ à esquerda. Realizada infusão de soro fisiológico por via abdominal em região de fistula, com posterior borbulhamento de dreno torácico, confirmando fistula pancreatopleural. Procedimento cirúrgico realizado sem intercorrências, paciente manteve-se estável, sem necessidade de uso de drogas vasoativas. O anatomopatológico concluiu pseudocisto pancreático com extensas áreas de fibrose e foram avaliados nove linfonodos, compativeis com hiperplasia linfoide reacional (Figura 3).

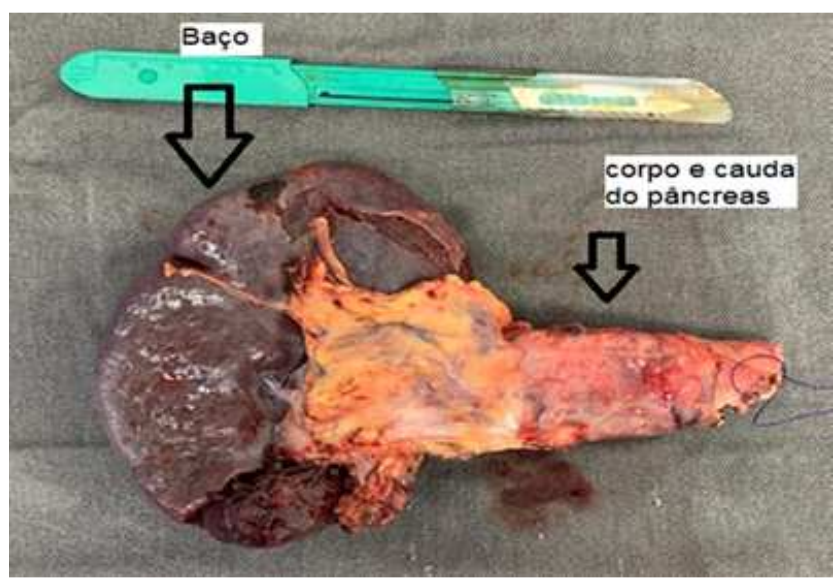

Figura 3: Peça cirúrgica produto de pancreatectomia corpocaudal + esplenectomia.

Em pós-operatório, coletado amilase de dreno abdominal e de dreno torácico, com valores decrescentes no primeiro, terceiro e sétimo dias, afastando fístula pancreática pós-operatória ou demais complicações cirúrgicas.

Apesar da drenagem pleural efetiva no primeiro momento, paciente manteve espessamento pleural em tomografia de tórax de controle, com diminuição de expansibilidade pulmonar em hemitórax esquerdo. Assim, após resolução do quadro abdominal, foi submetida a decorticação pulmonar esquerda por videotoracoscopia pela equipe de cirurgia torácica, em 10/02/2021.

Após 25 dias de hospitalização, em 20/02/2021, evoluiu com melhora completa do quadro respiratório e abdominal e teve alta hospitalar.

Paciente retornou em consulta ambulatorial com a equipe de cirurgia do aparelho digestivo em 26/03/2021 e com cirurgia torácica em 15/04/2021, estável e sem queixas, referindo melhora completa do 
quadro. Atualmente, segue em acompanhamento ambulatorial.

\section{DISCUSSÃO}

A fistula pancreatopleural pode ocorrer a partir da drenagem posterior do ducto pancreático principal em comunicação com a pleura; ou após a formação de um pseudocisto que se comunica com a cavidade pleural, seja ele roto ou não ${ }^{4}$.

O líquido pancreático penetra a cavidade pleural através do espaço retroperitoneal, adentrando o tórax, em geral, através do hiato esofageano, podendo ter continuidade com pericárdio, árvore brônquica ou esôfago, sendo a comunicação transdiafragmática a mais rara ${ }^{4}$.

$\mathrm{Na}$ grande maioria dos casos relatados, está mais associado a casos de pancreatite alcóolica, que corresponde a aproximadamente $84,37 \%$. As demais etiologias podem ser por pancreatite biliar $(9,37 \%)$, traumática $(3,12 \%)$ ou idiopática $(3,12 \%)^{5}$.

No relato em questão, a paciente não possuía histórico prévio de pancreatite, no entanto havia relato de agressão física constante por violência doméstica, motivo pelo qual foi descoberta a existência de um pseudocisto pancreático oito anos antes da formação da fístula pancreatopleural. Assim, sua etiologia traumática e a presença de comunicação através de trajeto bem delimitado transdiafragmático posterior corroboram ainda mais para a peculiaridade do caso.

A sintomatologia é geralmente frustra, com relato de dor abdominal em apenas 8\% dos casos. Quando ocorrem sintomas, estes estão associados ao quadro pulmonar, levando o paciente a procurar serviço médico por dispneia progressiva, como ocorreu no relato em questão. Pode estar associado também a anorexia, disfagia ou perda de peso ${ }^{5}$.

O derrame pleural pode ser bilateral ou unilateral, sendo mais frequente $o$ derrame pleural unilateral à esquerda, em $48 \%$ dos casos. $\mathrm{Na}$ análise do líquido pleural, encontra-se nível elevado de amilase, sendo os demais parâmetros em geral normais ${ }^{5}$.

Neste caso, a paciente apresentou derrame pleural unilateral à esquerda e análise de liquido pleural com amilase elevada, sem demais alterações nos parâmetros bioquímicos do líquido pleural, compativel com a epidemiologia apresentada na literatura.

Para o diagnóstico, o melhor exame é a ressonância magnética com pancreatografia, que possibilita a visualização da fístula e suas relações anatômicas ${ }^{5}$.

A colangiopancreatografia retrógrada endoscópica também pode ser realizada como método diagnóstico, porém é mais invasiva e em geral direcionada a casos em que será feita proposta terapêutica, como a passagem de stent pancreático ${ }^{4,7}$.

Por se tratar de uma patologia rara, não existem ensaios clínicos randomizados que proponham uma terapêutica única, sendo assim o tratamento baseado nos relatos de casos descritos na literatura e na experiência do profissional.

Para casos com fistulas de menor débito, sem ruptura pancreática, pode ser realizado o tratamento clínico. Consiste na realização de toracocentese de alivio ou drenagem torácica, jejum via oral, nutrição parenteral total e uso de somatostatina ou octreotide por três semanas, com o intuito de diminuir a secreção pancreática. Há resolução com medidas clínicas em torno de $45 \%$ dos pacientes, dispensando demais terapias ${ }^{4}$.

Além disso, pode ser realizada terapêutica alternativa endoscópica com passagem de stent ou de sonda nasopancreática no ducto pancreático principal para drenagem de suco pancreático, sendo que esta deve ser mantida por até dois meses. Já em relação ao stent, não há consenso e depende da experiência do endoscopista. Tal procedimento tem mostrado bons resultados na literatura, dispensando o tratamento cirúrgico em até $25 \%$ dos $\operatorname{casos}^{7}$.

Em pacientes com rotura do pseudocisto, fístulas de grande volume ou intratabilidade clínica, é necessária a abordagem cirúrgica, a qual depende da localização do trajeto fistuloso6,8.

Se o trajeto da fistula apresentar-se na cauda pancreática, o paciente será submetido a uma pancreatectomia distal. Caso esteja na cabeça do pâncreas, será necessária a realização de uma duodenopancreatectomia. Ambos os procedimentos são efetivos em $80 \%$ dos casos, com taxa de mortalidade de $4,5 \%{ }^{5,8}$. 
Neste relato, a paciente estava em programação cirúrgica para correção de pseudocisto, quando apresentou sintomatologia súbita de dispneia com derrame pleural e formação de fistula pancreatopleural de grande volume. Foi submetida a drenagem pleural, e por conta da fistula e do alto débito diário em dreno torácico, optou-se pela terapia cirúrgica.

Além disso, devido ao espessamento pleural formado pela reação inflamatória do suco pancreático, teve também que ser submetida a uma decorticação pleural por videtoracoscopia à esquerda. Ambos os procedimentos foram realizados com sucesso, sem complicações pós-operatórias.

Dessa forma, o caso em questão mostra um tratamento cirúrgico efetivo de uma fistula pancreatopleural por um pseudocisto de padrão incomum, trazendo peculiaridade em seu relato.

\section{CONCLUSÃo}

A fistula pancreatopleural é uma complicação rara de casos pancreatite, com poucos relatos na literatura.

O relato em questão traz uma evolução incomum de um pseudocisto traumático com fistula pancreatopleural transdiafragmática, na qual foi realizado procedimento cirúrgico com sucesso.

\section{REFERÊNCIAS}

1. Costa OL, Zago-Gomes MP, Gonçalves C. Pancreatite aguda grave: resultado do tratamento de 68 pacientes. Rev. Col. Bras. Cir. 2004;31(2):112-6.

doi: $\quad 10.1590 /$ S010069912004000200008.

2. Abreu R, Carvalho-Jr R, Vaz F, Ota L, Speranzini M. Drenagem endoscópica transmural de pseudocisto pancreático: resultados a longo prazo. Arq. Gastroenterol. 2007;44(1):29-34. doi: 28032007000100007.

$10.1590 / \mathrm{S} 0004-$

3. Banks PA, Bollen TL, Dervenis C, et al. Classification of acute pancreatitis 2012: revision of the Atlanta classification and definitions by international consensus. Gut. 2013;62(1):102-11. doi:
4. Cazzo E, Apodaca-Rueda M, Gestic M, Chaim F, Saito $H$, Utrini $M$, et al. Management of pancreaticopleural fistulas secondary to chronic pancreatitis. ABCD arq. bras. cir. dig. 2017;30(3):225-8. doi: 10.1590/0102-6720201700030014.

5. Zurriaebe L, Oyarzabal I, Beguiristains A, Amato E, Zapata E, Salvador P. Fístula pancreaticopleural: pruebas diagnosticas y tratamento. Cir Esp. 2005;77(6):359-61. doi: 10.1016/S0009-739X(05)70871-X.

6. Vyas S, Gogoi D, Sinha S, Singh P, Yadav T, Khandelwal N. Pancreaticopleural fistula: an unusual complication of pancreatitis diagnosed with magnetic resonance cholangiopancreatography. JOP. 2009;10(6):6713.

7. Mota R, Alcântara C, Antunes L, Jorge A. Pancreaticopleural fistula. ABCD arq. bras. cir. dig. 2011;24(3):251-2. doi: $10.1590 /$ S010267202011000300016.

8. Aswani Y, Hira P. Pancreaticopleural fistula: a review. JOP. 2015;16(1):904. doi: 10.6092/1590-8577/2915.
Fonte de financiamento: Não

Conflito de interesses: Não

Data de Submissão: 03 Maio 2021

Decisão final: 19 Setembro 2021

\section{Autor de Correspondência:}

Gabriela Camilo Teixeira

E-mail: gabriela.teixeira13@gmail.com 